\title{
Regional Heart Motion Abnormality Detection via Information Measures and Unscented Kalman Filtering
}

\author{
Kumaradevan Punithakumar ${ }^{1}$, Ismail Ben Ayed ${ }^{1}$, Ali Islam², \\ Ian G. Ross ${ }^{3}$, and Shuo $\mathrm{Li}^{1,4}$ \\ 1 GE Healthcare, London, Ontario, Canada \\ 2 St. Joseph's Health Care, London, Ontario, Canada \\ 3 London Health Science Center, London, Ontario, Canada \\ 4 University of Western Ontario
}

\begin{abstract}
This study investigates regional heart motion abnormality detection using various classifier features with Shannon's Differential Entropy (SDE). Rather than relying on elementary measurements or a fixed set of moments, the SDE measures global distribution information and, as such, has more discriminative power in classifying distributions. Based on functional images, which are subject to noise and segmentation inaccuracies, heart wall motion analysis is acknowledged as a difficult problem and, therefore, incorporation of prior knowledge is desirable to enhance the accuracy. Given noisy data and nonlinear dynamic model to describe the myocardial motion, unscented Kalman filter, a recursive nonlinear Bayesian filter, is devised in this study so as to estimate LV cavity points. Subsequently, a naive Bayes classifier algorithm is constructed from the SDEs of different features in order to automatically detect abnormal functional regions of the myocardium. Using $90 \times 20$ segmented LV cavities of short-axis magnetic resonance images obtained from 30 subjects, the experimental analysis carried over 480 myocardial segments demonstrates that the proposed method perform significantly better than other recent methods, and can lead to a promising diagnostic support tool to assist clinicians.
\end{abstract}

\section{Introduction}

Assessment of left ventricular function is of utmost importance in the diagnosis of coronary heart disease, the leading cause of death worldwide. It primarily relies on the visual analysis and interpretation of wall motion and, thus, subject to high interobserver variability. Alternatively, computer-aided detection systems have been attempted in recent years in order to automatically analyze the wall motion quantitatively 123 , and to classify hearts into normal or abnormal 456. However, in clinical practice, the wall motion is commonly scored by following a standard issued by American Heart Association (AHA) 7], where the myocardium is divided into 17 segments. Therefore, regional abnormality analysis is more desirable for clinical purposes. Existing heart motion regional 
analysis methods are based on, among others, shape models with localized variations [8], a tensor-based classification to conserve the spatiotemporal structure of the myocardium deformation 9], a hidden Markov model for local wall motion classification based on stress echocardiography [10, a pattern recognition method based on intra-segment correlation [11], and an independent component analysis classifier to detect and localize abnormally contracting regions [12. Most of existing methods either suffer from poor accuracy, use data that are not available in regular clinical routine, or require extensive user interaction to define myocardial boundaries.

Nevertheless, automating abnormality analysis would reduce inter- and intraobserver variability and, therefore, subjectivity in the analysis. However, it requires precise estimation of the myocardial points. As such, accurate characterization of dynamic behavior of Left Ventricle (LV) is essential in order to enhance the performance of motion estimation. In this connection, linear dynamic models may not be sufficient to describe the LV dynamics and, therefore, a nonlinear cyclic model is proposed in this study. The proposed model consists of a time-varying parameter, angular frequency, to be estimated from the data along with other state elements. This is achieved by augmenting the angular frequency with other state elements, and by estimating the augmented state using Unscented Kalman Filter (UKF), a recursive nonlinear Bayesian filter, given initial LV cavity points and a nonlinear cyclic model.

Cine Magnetic Resonance (MR) sequences are widely used for cardiac functional analysis, and provide a large number of image: 1 . Therefore, tracking based on manual delineation of the LV boundary in all these images is prohibitively time consuming. Alternatively, automating the process can be of great interest [13 14. However, it is subject to the difficulties due to the low contrast and photometric similarities between the connected cardiac regions - for instance, the papillary muscles within the cavity and myocardial wall have approximately the same intensity. To tackle the problem of obtaining initial LV segmentations, this study adopts a non-rigid image registration method that minimizes the sum of squared intensity differences 15, given an initial segmentation of the first frame. Rather than using a segmentation algorithm to obtain initial LV cavity points, using registration is advantageous in our study as it provides the sequence of corresponding points over time, an essential attribute to analyze wall motion regionally.

Classification of regional abnormality is a difficult problem due to the similarity between the statistical information associated with normal and abnormal heart motions. In this study, we investigate the problem with a global measure based on the Shannon's Differential Entropy (SDE) of the distributions of normalized radial distance, radial velocity, segment area and circumferential length of a segment. Rather than relying on elementary measurements or a fixed set of moments, the SDE measures global distribution information and, as such, has more discriminative power in classifying distributions. The individual classification ability of elements were measured using Receiver Operating Characteristic (ROC) curves with

${ }^{1}$ Typically, the number of images per subject is equal to 200 . 
the corresponding Area Under the Curves (AUCs), and the Bhattacharyya distance metric [16]. Subsequently, a naive Bayes classifier algorithm is constructed from the SDEs of classifier elements with the best performance in order to automatically detect abnormal functional regions of the myocardium.

Using 90 image datasets, each consisting of 20 segmented LV cavities of shortaxis MR functional images obtained from 30 subjects, the proposed method is quantitatively evaluated by comparison with ground truth classifications by radiologists over 480 myocardial segments. The proposed method performed significantly better than other recent methods with an overall classification accuracy of $91.0 \%$, and can lead to a promising diagnostic support tool to assists clinicians.

\section{Temporal Smoothing of the Dataset}

Dynamic Model for Temporal Periodicity: Let $(x, y)$ be a point on the boundary of the $L V$ cavity and $\mathbf{s}=[\bar{x} \times \dot{x} \bar{y} \text { y } \dot{y} \omega]^{T}$ be the state vector that describes the corresponding dynamics. The elements $\dot{x}, \bar{x}, \dot{y}$ and $\bar{y}$ denote, respectively, velocity and the mean position over a cardiac cycle in $\mathrm{x}$ and $\mathrm{y}$ coordinate directions, and $\omega$ the angular frequency. We assume that the heart motion is periodic. The discrete-time dynamic model that describes the cyclic motion of the point is given by

$$
\mathbf{s}_{k+1}=F_{k} \mathbf{s}_{k}+v_{k}
$$

where

$$
F_{k}=\left[\begin{array}{ccc}
F_{c y}(k) & \mathbf{0} & \mathbf{0} \\
\mathbf{0} & F_{c y}(k) & \mathbf{0} \\
\mathbf{0} & \mathbf{0} & 1
\end{array}\right], F_{c y}(k)=\left[\begin{array}{rrr}
1 & 0 & 0 \\
1-\cos \left(\omega_{k} T\right) & \cos \left(\omega_{k} T\right) & \frac{1}{\omega_{k}} \sin \left(\omega_{k} T\right) \\
\omega_{k} \sin \left(\omega_{k} T\right) & -\omega_{k} \sin \left(\omega_{k} T\right) & \cos \left(\omega_{k} T\right)
\end{array}\right] \text {, }
$$

and $T$ is the time interval between two subsequent image frames. The process noise sequence $\left\{v_{k}\right\}$ is Gaussian with zero-mean and covariance $Q_{k}$ that accommodates the unpredictable errors due to modeling uncertainties. The measurement equation is given by

$$
z_{k}=H_{k} \mathbf{s}_{k}+\eta_{k}
$$

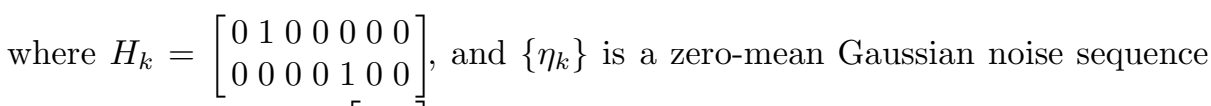
with covariance $R_{k}=\left[\begin{array}{ll}r & 0 \\ 0 & r\end{array}\right]$.

Unscented Kalman Filter: The dynamic model in (1) is nonlinear and, therefore, we adopt a recursive nonlinear Bayesian filter, the UKF [17, to estimate the state $s_{k}$ at each time step. The UKF uses unscented transformation, a more direct and explicit mechanism for transforming mean and covariance information, that addresses the deficiencies of linearization inherent to other adaptive filters such as Extended Kalman Filter (EKF). UKF propagates the second order properties of the distribution with only a small amount of statistical information by choosing sample points deterministically. Therefore, it provides sufficient accuracy for nonlinear filtering applications with computational cost of order similar to the EKF. 


\section{Information Theoretic Measures and Classifier}

The state estimates from UKF are subsequently processed towards the classification of normal or abnormal motions. In order to measure the information associated with regional LV function, the SDEs of a set of classifier elements were evaluated.

Classifier elements: Let $\hat{\mathbf{s}}_{k, i}=\left[\hat{\overline{\mathrm{x}}}_{k, i} \hat{\mathrm{x}}_{k, i} \hat{\dot{\mathrm{x}}}_{k, i} \quad \hat{\overline{\mathrm{y}}}_{k, i} \hat{\mathrm{y}}_{k, i} \hat{\dot{\mathrm{y}}}_{k, i} \hat{\omega}_{k, i}\right]^{T}$ be the estimated state of $i^{\text {th }}$ point by UKF at time step $k$. Let $I_{N}=\{1,2, \ldots, N\}$ and $I_{S}=\left\{i_{s}, i_{s}+1, \ldots, i_{s}+N_{s}-1\right\} \subset I_{N}$ be the set of points on the LV cavity and segment $S$, respectively. The center $\left(c_{\mathrm{x}, k}, c_{\mathrm{y}, k}\right)$ of the LV cavity at time step $k$ is given by $c_{\mathrm{x}, k}=\frac{1}{N} \sum_{i \in I_{N}} \hat{\mathrm{x}}_{k, i}$ and $c_{\mathrm{y}, k}=\frac{1}{N} \sum_{i \in I_{N}} \hat{\mathrm{y}}_{k, i}$. We propose to use the following classifier elements.

1. Normalized radial distance

$$
r_{k, i}=\frac{\sqrt{\left(\hat{\mathrm{x}}_{k, i}-c_{\mathrm{x}, k}\right)^{2}+\left(\hat{\mathrm{y}}_{k, i}-c_{\mathrm{y}, k}\right)^{2}}}{\max _{i \in I_{N}} \sqrt{\left(\hat{\mathrm{x}}_{k, i}-c_{\mathrm{x}, k}\right)^{2}+\left(\hat{\mathrm{y}}_{k, i}-c_{\mathrm{y}, k}\right)^{2}}} \quad \forall i \in I_{S}
$$

2. Radial velocity

$$
v_{k, i}=\left\langle\boldsymbol{v}_{k, i} \cdot \frac{\boldsymbol{r}_{k, i}}{\left\|\boldsymbol{r}_{k, i}\right\|}\right\rangle \quad \forall i \in I_{S}
$$

where $\boldsymbol{v}_{k, i}=\left[\hat{\dot{\mathrm{x}}}_{k, i}, \hat{\dot{\mathrm{y}}}_{k, i}\right]^{T}$ and $\boldsymbol{r}_{k, i}=\left[\hat{\mathrm{x}}_{k, i}-c_{\mathrm{x}, k}, \hat{\mathrm{y}}_{k, i}-c_{\mathrm{y}, k}\right]^{T}$

3. Circumferential length

$$
l_{k}=\sum_{i=i_{s}}^{i_{s}+N_{s}-2} \sqrt{\left(\hat{\mathrm{x}}_{k, i+1}-\hat{\mathrm{x}}_{k, i}\right)^{2}+\left(\hat{\mathrm{y}}_{k, i+1}-\hat{\mathrm{y}}_{k, i}\right)^{2}}
$$

4. Segment area

$$
\begin{array}{r}
a_{k}=\frac{1}{2}\left[\left(c_{\mathrm{x}, k} \hat{\mathrm{y}}_{k, i_{s}}-c_{\mathrm{y}, k} \hat{\mathrm{x}}_{k, i_{s}}\right)+\left(\hat{\mathrm{x}}_{k, i_{s}+N_{s}} c_{\mathrm{y}, k}-\hat{\mathrm{y}}_{k, i_{s}+N_{s}} c_{\mathrm{x}, k}\right)\right. \\
\left.+\sum_{i=i_{s}}^{i_{s}+N_{s}-2}\left(\hat{\mathrm{x}}_{k, i} \hat{\mathrm{y}}_{k, i+1}-\hat{\mathrm{x}}_{k, i+1} \hat{\mathrm{y}}_{k, i}\right)\right]
\end{array}
$$

Shannon's differential entropy and Bayesian classifier: The kernel density estimate of a classifier element $\chi_{n} \in\left\{r_{k, i}, v_{k, i}, l_{k}, a_{k}\right\}$ is given by

$$
f(\xi)=\frac{\sum_{n=1}^{n_{\chi}} \mathcal{K}\left(\chi_{n}-\xi\right)}{n_{\chi}}
$$

where $n_{\chi}=K N_{s}$ for $\chi_{n} \in\left\{r_{k, i}, v_{k, i}\right\}$ and $n_{\chi}=K$ for $\chi_{n} \in\left\{l_{k}, a_{k}\right\}$. Typical choices of $\mathcal{K}(\cdot)$ are the Dirac function and the Gaussian kernel. In this study, the SDE is derived as follows

$$
S_{f}=-\int_{\xi \in \mathbb{R}} \frac{\sum \mathcal{K}\left(\chi_{n}-\xi\right)}{n_{\chi}}\left(\ln \sum \mathcal{K}\left(\chi_{n}-\xi\right)-\ln n_{\chi}\right) d \xi
$$


It is advantageous to use multiple SDE measures towards the classification as they measure different information associated with the myocardial function. A naive Bayesian classifier that provides a quadratic decision boundary is constructed from SDEs of classifier elements.

\section{Experiments}

The data contains 90 short-axis image datasets, each consisting of 20 functional 2D images acquired from 20 normal and 10 abnormal hearts. The data were acquired on 1.5T MRI scanners with fast imaging employing steady state acquisition (FIESTA) mode. The results of 480 myocardial segments from apical, mid-cavity and basal frames were compared with ground truth classification of the cine MRI datasets by radiologist:2. The dynamic model and UKF parameters are chosen as $q_{1}=0.01, q_{2}=0.1 q_{3}=1$ and $r=0.01$ to accommodate noise that account for modeling uncertainties. We used Dirac function for $\mathcal{K}(\cdot)$ in the kernel density estimation.

In Fig. 1, we give a representative sample of the segmentation results for apical, mid-cavity and basal frames. The frames were automatically segmented following the standard issued by the AHA [7, given anatomical landmarks 3 on the first frame.

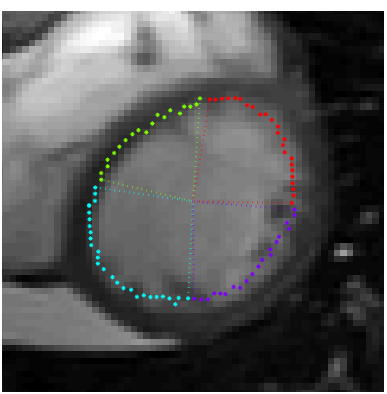

(a) Apical

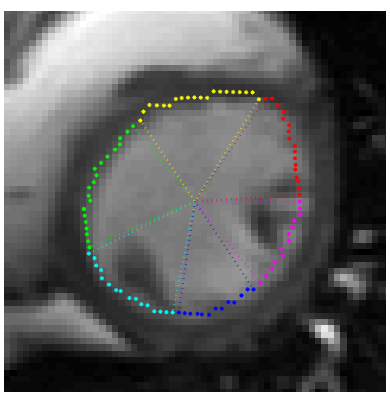

(b) Mid-cavity

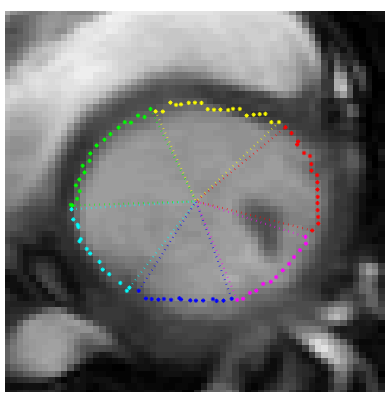

(c) Basal

Fig. 1. Representative examples of segmented myocardium using the proposed approach. Apical, mid-cavity and basal frames were segmented, respectively, into 4, 6 and 6 segments following the standard in 7 .

We used two criteria to measure the performance of each classifier elements, namely, the ROC curves with corresponding AUCs, and Bhattacharyya measure [16] to assess the discriminative power of each classifier elements. Furthermore, we assessed the performance of the proposed approach via leave-one-subject-out method.

${ }^{2}$ Among the 480 myocardial segments, 389 segments were marked as normal and 91 as abnormal.

${ }^{3}$ As suggested by [7, the attachment of the right ventricular wall to the LV is used to identify and separate the septum from the LV anterior and inferior free walls. 
ROC, AUC and Bhattacharyya measure: The ROC curves for classifier elements is shown in Fig. 2. The figure shows that SDEs of segment area and normalized radial distance have better classifying ability than other classifier elements. The AUCs corresponding to the ROC curves in Fig. 2 are reported in Table 1. We used the Bhattacharyya distance metric to evaluate the overlap between the distributions of classifier elements over normal and abnormal motions. The SDEs of segment area and normalized radial distance yielded the higher $\mathcal{B}$ as reported in Table 1 and, therefore, have the best discriminative ability. This is consistent with the previous findings based on ROC/AUC evaluations.

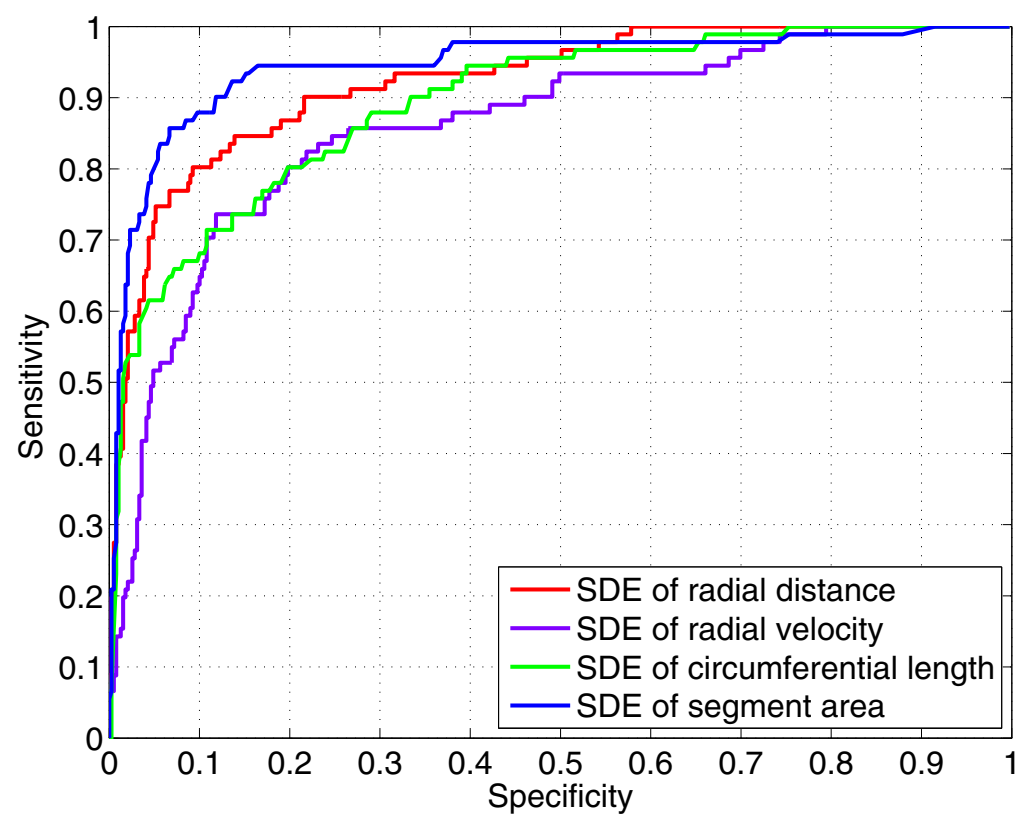

Fig. 2. Receiver operating characteristics of classifier elements. The closer the curve to the left hand top corner, the better the classification performance.

Table 1. The area under the curve corresponding to Fig. 2 and the Bhattacharyya distance metric $(\mathcal{B})$ of normal/abnormal distributions. The higher the values the better the discriminative ability of the classifier.

Classifier element

SDE of segment area

SDE of normalized radial distance

SDE of circumferential shrink

SDE of radial velocity
Bhattacharyya distance AUC $(\%) \quad \operatorname{metric}(\mathcal{B})$

$\begin{array}{ll}94.3 & 0.66 \\ 92.1 & 0.61 \\ 89.0 & 0.53 \\ 85.8 & 0.48\end{array}$


Table 2. The percentage of classification accuracy using leaving-one-subject-out method. The proposed method achieved an overall classification accuracy of $90.8 \%$.

\begin{tabular}{lccc}
\hline & Accuracy $(\%)$ & Sensitivity $(\%)$ & Specificity (\%) \\
\hline Apex & 92.5 & 90.0 & 93.3 \\
Mid-cavity & 93.3 & 93.1 & 94.1 \\
Base & 87.2 & 100.0 & 84.9 \\
\hline
\end{tabular}

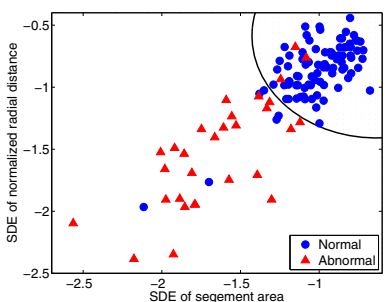

(a) Apical

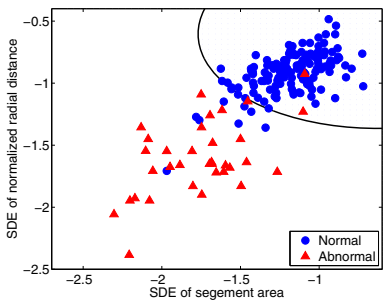

(b) Mid-cavity

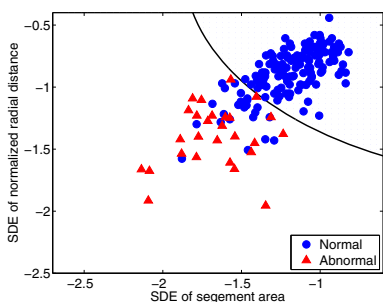

(c) Basal

Fig. 3. Decision boundary for normal and abnormal regional myocardial functions using a Bayesian classifier

Classification performance: Table 2 reports the classification performance of correctly classified hearts using a leaving-one-subject-out method. A naive Bayes classifier algorithm is constructed from the SDEs of the segment area and normalized radial distance, the elements with better classifier ability. Fig. 3 shows the quadratic decision boundary for normal/abnormal classification, where blue circles represent the normal function and red triangles the abnormal. The overall classification accuracy is equal to $90.8 \%$, with a sensitivity of $94.5 \%$ and specificity of $90.0 \%$. The highest performance was achieved for mid-cavity frames with average of $93.3 \%$ for accuracy, $93.1 \%$ for sensitivity and $94.1 \%$ for specificity.

\section{Conclusions}

We presented a regional heart motion abnormality detection method using the SDE and UKF. A non-rigid image registration method was adopted to initialize the LV cavity points, and the results were subsequently processed using UKF, given a nonlinear dynamic model. The myocardial segments were identified following the standard issued by AHA. The SDE of normalized radial distance, radial velocity, segment area and circumferential segment length were evaluated for each myocardial segments and a naive Bayesian classifier is constructed from the SDEs. The experimental analysis carried over $90 \times 20$ segmented LV cavities of short-axis MR images obtained from 30 subjects demonstrates that the proposed method perform significantly better than other recent methods, and can lead to a promising diagnostic support tool to assists clinicians. 


\section{References}

1. McEachen, J., Nehorai, A., Duncan, J.: Multiframe temporal estimation of cardiac nonrigid motion. IEEE Trans. Image Process. 9(4), 651-665 (2000)

2. Spottiswoode, B., Zhong, X., Hess, A., Kramer, C., Meintjes, E., Mayosi, B., Epstein, F.: Tracking myocardial motion from cine dense images using spatiotemporal phase unwrapping and temporal fitting. IEEE Trans. Med. Imag. 26(1), 15-30 (2007)

3. Sundar, H., Davatzikos, C., Biros, G.: Biomechanically-constrained 4D estimation of myocardial motion. In: Yang, G.-Z., Hawkes, D., Rueckert, D., Noble, A., Taylor, C. (eds.) MICCAI 2009. LNCS, vol. 5762, pp. 257-265. Springer, Heidelberg (2009)

4. Punithakumar, K., Li, S., Ayed, I.B., Ross, I., Islam, A., Chong, J.: Heart motion abnormality detection via an information measure and bayesian filtering. In: Yang, G.-Z., Hawkes, D., Rueckert, D., Noble, A., Taylor, C. (eds.) MICCAI 2009. LNCS, vol. 5762, pp. 373-380. Springer, Heidelberg (2009)

5. Fung, G., Qazi, M., Krishnan, S., Bi, J., Rao, B., Katz, A.: Sparse classifiers for automated heartwall motion abnormality detection. In: Proc. IEEE 4th Int. Conf. Machine Learning and Applications, pp. 194-200 (2005)

6. Qazi, M., Fung, G., Krishnan, S., Bi, J., Bharat Rao, R., Katz, A.: Automated heart abnormality detection using sparse linear classifiers. IEEE Eng. Med. Biol. Mag. 26(2), 56-63 (2007)

7. Cerqueira, M.D., Weissman, N.J., Dilsizian, V., Jacobs, A.K., Kaul, S., Laskey, W.K., Pennell, D.J., Rumberger, J.A., Ryan, T., Verani, M.S.: Standardized myocardial segmentation and nomenclature for tomographic imaging of the heart: A statement for healthcare professionals from the cardiac imaging committee of the council on clinical cardiology of the American Heart Association. Circulation 105(4), 539-542 (2002)

8. Leung, K.Y., Bosch, J.G.: Localized shape variations for classifying wall motion in echocardiograms. In: Ayache, N., Ourselin, S., Maeder, A. (eds.) MICCAI 2007, Part I. LNCS, vol. 4791, pp. 52-59. Springer, Heidelberg (2007)

9. Mansor, S., Noble, J.: Local wall motion classification of stress echocardiography using a hidden Markov model approach. In: The 5th IEEE Int. Symp. Biomedical Imaging: From Nano to Macro, pp. 1295-1298 (2008)

10. Qian, Z., Liu, Q., Metaxas, D.N., Axel, L.: Identifying regional cardiac abnormalities from myocardial strains using spatio-temporal tensor analysis. In: Metaxas, D., Axel, L., Fichtinger, G., Székely, G. (eds.) MICCAI 2008, Part I. LNCS, vol. 5241, pp. 789-797. Springer, Heidelberg (2008)

11. Lu, Y., Radau, P., Connelly, K., Dick, A., Wright, G.: Pattern recognition of abnormal left ventricle wall motion in cardiac MR. In: Yang, G.G., Hawkes, D., Rueckert, D., Noble, A., Taylor, C. (eds.) MICCAI 2009. LNCS, vol. 5762, pp. 750-758. Springer, Heidelberg (2009)

12. Suinesiaputra, A., Frangi, A., Kaandorp, T., Lamb, H., Bax, J., Reiber, J., Lelieveldt, B.: Automated detection of regional wall motion abnormalities based on a statistical model applied to multislice short-axis cardiac MR images. IEEE Trans. Med. Imag. 28(4), 595-607 (2009)

13. Ben Ayed, I., Punithakumar, K., Li, S., Islam, A., Chong, J.: Left ventricle segmentation via graph cut distribution matching. In: Yang, G.Z., et al. (eds.) MICCAI 2009. LNCS, vol. 5762, pp. 901-909. Springer, Heidelberg (2009) 
14. Ben Ayed, I., Lu, Y., Li, S., Ross, I.: Left ventricle tracking using overlap priors. In: Metaxas, D., Axel, L., Fichtinger, G., Székely, G. (eds.) MICCAI 2008, Part I. LNCS, vol. 5241, pp. 1025-1033. Springer, Heidelberg (2008)

15. Chen, H.M., Ben Ayed, I., Garvin, G., Goela, A., Li, S.: A parameterization of deformation fields for diffeomorphic image registration and its application to myocardial delineation. In: MICCAI 2010 (2010) (accepted for publication)

16. Comaniciu, D., Ramesh, V., Meer, P.: Kernel-based object tracking. IEEE Trans. Pattern Anal. Mach. Intell. 25(5), 564-577 (2003)

17. Julier, S., Uhlmann, J.: Unscented filtering and nonlinear estimation. Proc. IEEE 92(3), 401-422 (2004) 